\title{
No treatment for asymptomatic common bile ducts stones?
}

\section{다 (용}

\author{
Author \\ Pier Alberto Testoni \\ Institution \\ Division of Gastroenterology and Gastrointestinal \\ Endoscopy, Vita-Salute San Raffaele University, Milan \\ Bibliography \\ DOI https://doi.org/10.1055/s-0043-107778 | \\ Endoscopy International Open 2017; 05: E1151-E1152 \\ (c) Georg Thieme Verlag KG Stuttgart · New York \\ ISSN 2364-3722
}

\author{
Corresponding author \\ Pier Alberto Testoni, MD, Vita-Salute San Raffaele \\ University, San Raffaele Scientific Institute, Division of \\ Gastroenterology and Gastrointestinal Endoscopy, Milano, \\ Lombardia, Italy \\ testoni.pieralberto@hsr.it
}

Common bile stone disease is currently treated by therapeutic endoscopic retrograde cholangiopancreatography (ERCP), and no one doubts the need to remove the stones as soon as possible, regardless of symptoms reported by the patient, and as recommended in all available guidelines [1 - 3]. The indication for treatment derives from the risk of potentially severe wellknown complications related to stones retained in the common bile duct, such as acute biliary pancreatitis and cholangitis. These complications may occur if a stone impacts at the level of the pancreatico-biliary junction and impaction can occur abruptly even after a long period of clinically silent history.

Complications related to migration of stones into the common bile duct (CBD) have been estimated to occur in about 25 $\%$ of cases of asymptomatic gallstone disease over a follow-up period of 10 years [4]. However, we don't know whether this complication rate may be even higher in the presence of asymptomatic CBD disease, since the natural history of the condition is still unclear, depending on the lack of long-term follow-up of untreated cases. Data obtained in patients undergoing laparoscopic cholecystectomy with residual CBD stones showed that in about one-third of cases stones passed spontaneously within 6 weeks after intervention [5].

In fact, to date, no one in clinical practice has questioned whether ERCP is really better and safer than no treatment in cases of asymptomatic CBD stone disease in patients who would be candidates for the procedure. None of us have balanced the risk of inducing an acute complication - sometimes life-threatening - by treating an asymptomatic CBD stone versus the risk of complication related to the natural history of the disease.

To my knowledge, Saito et al are the first to assess outcomes of therapeutic ERCP in asymptomatic patients with CBD stones, in a retrospective multicenter study carried out on a series of 425 consecutive asymptomatic or symptomatic patients undergoing ERCP for stone removal [6]. These authors should be commended for addressing this still unsettled point.

They found that the complication rate after ERCP was higher in asymptomatic subjects compared with symptomatic ones (26.9\% vs 3.3\%). Moderate or severe complications were also more frequent in asymptomatic subjects. They also found that complications related to endoscopic stone removal were more severe in elderly patients, the very patients who probably are less prone to have complications or suffer from pain in the natural history of the disease.

Post-ERCP pancreatitis, the most frequent and feared complication associated with ERCP procedures, occurred 7 to 8 times more frequently in asymptomatic subjects compared with symptomatic ones ( $16.4 \%$ vs $2.2 \%, P<0.0001)$. Other less common complications, such as cholangitis and perforation, occurred more frequently in asymptomatic subjects, too, although the difference was not significant.

The authors also performed univariate and multivariate analyses of risk factors for post-ERCP pancreatitis in their series. Seven factors were found significant in the univariate analysis: indication for ERCP (asymptomatic CBD stone disease), prolonged (>10 min) cannulation time, technique for sphincterotomy, normal serum bilirubin level, biliary stent placement (no), pancreatic stent placement (yes), and antibiotics use (no). Interestingly and differently from others studies assessing the risk factors for post-ERCP pancreatitis [7-12] the presence of a non-dilated CBD was not considered in this multivariate analysis. 
More interestingly, in multivariate analysis, only the indication for ERCP (asymptomatic subjects) appeared to be a significant risk factor for post-ERCP pancreatitis.

Why might therapeutic ERCP be more dangerous in asymptomatic CBD stone disease than in symptomatic disease? Very likely, in asymptomatic cases the CBD tends to be non-dilated and the papillary orifice small because of the low bile duct pressure, in the absence of cholestasis. Therefore, most of these patients have several risk factors for post-ERCP pancreatitis compared with symptomatic patients, both patient- and techniquerelated, independent of presence of intraductal stones, such as presence of a non-dilated $C D$, the absence of cholestasis, and a small papillary orifice which result in difficult cannulation with prolonged cannulation time and an increased number of attempts at cannulating [13]. On the basis of these considerations (and according to my personal experience), asymptomatic patients with a dilated CBD have a post-ERCP risk of developing pancreatitis that is similar to that reported for symptomatic patients and standard-risk subjects. Reading the paper by Saito et al, I believe that our perspective on ERCP for CBD stone disease needs to change. Patients with $C B D$ stone disease with a non-dilated CBD, normal bilirubin levels, and small papilla have a higher risk of post-ERCP complications (mainly pancreatitis) compared to patients with dilated CBD stone disease, high bilirubin level, and prominent papilla. The first condition is more frequently seen in asymptomatic patients, so they have a higher incidence of post-ERCP complications. On the other hand, other therapeutic approaches different from ERCP are not to be proposed in these patients, because they are associated with higher morbidity.

In conclusion, comparing risks related to therapeutic ERCP and no treatment, we have to consider a $27 \%$ risk of complications associated with endoscopic therapy (reported by this study) and an average $30 \%$ risk of complications reported in the natural history of CBD stone disease. The overall risk of complications does not differ substantially between the 2 strategies.

While we await for further studies in larger series of patients, I believe that we should follow the current guidelines and treat all patients with CBD stones, independent of symptoms. However, when talking to patients with asymptomatic CBD stone disease who have conditions that place them a high risk of post-ERCPO complications, we need to take into account the data reported by Saito et al. and the available studies on risk factors for post-ERCP complications and discus with them risks and benefits of endoscopic treatment versus a "wait and see" strategy.
Competing interests

None

References

[1] Williams EJ, Green J, Beckingham I et al. Guidelines on the management of common bile duct stones (CBDS). Gut 2008; 57: 1004-1021

[2] Maple JT, Ikenberry SO, Anderson MA et al. Gastrointest Endosc 2011; 74: $731-744$

[3] Tazuma S, Unno M, Igarashi Y et al. Evidence-based clinical practice guidelines for cholelithiasis. J Gastroenterol 2017; 52: 276-300

[4] Johnson AG, Hosking SW. Appraisal of the management of bile duct stones. Br J Surg 1987; 74: 555 - 560

[5] Collins C, Maguire D, Ireland A et al. A prospective study of common bile duct calculi in patients undergoing laparoscopic cholecystectomy: natural history of choledocholithiais revisited. Ann Surg 2004; 239: $28-33$

[6] Saito H, Kumamoto CB, Kakuma T et al. Increased risk and severity of ERCP-related complications associated with asymptomatic common bile duct stones. Endoscopy International Open 2017; 05: 809-817

[7] Masci E, Mariani A, Curioni S et al. Risk factors for pancreatitis following endoscopic retrograde cholangiopancreatography: a meta-analysis. Endoscopy 2003; 35: $830-834$

[8] Freeman ML, DiSario JA, Nelson DB et al. Risk factors for post-ERCP pancreatitis: a prospective multicenter study. Gastrointest Endosc 2001; 54: $425-434$

[9] Dumonceau JM, Andriulli A, Elmunzer BJ et al. Prophylaxis of postERCP pancreatitis: European Society of Gastrointestinal Endoscopy (ESGE) Guideline - updated June 2014. Endoscopy 2014; 46: 799 815

[10] Chen J], Wang XM, Liu XQ et al. Risk factors for post-ERCP pancreatitis: a systematic review of clinical trials with a large sample size in the past 10 years. Eur J Med Res 2014; 19: 26

[11] Ding X, Zhang F, Wang Y. Risk factors for post-ERCP pancreatitis: A systematic review and meta-analysis. Surgeon 2015; 13: 218-229

[12] El Nakeeb A, El Hanafy E, Salah T et al. Post-endoscopic retrograde cholangiopancreatography pancreatitis: Risk factors and predictors of severity. World J Gastrointest Endosc 2016; 8: 709-715

[13] Testoni PA, Mariani A, Aabakken L et al. Papillary cannulation and sphincterotomy techniques at ERCP: European Society of Gastrointestinal Endoscopy (ESGE) Clinical Guideline. Endoscopy 2016; 48: $657-683$ 